Scientific journal

PHYSICAL AND MATHEMATICAL EDUCATION

Has been issued since 2013.

Науковий журнал

ФІЗИКО-МАТЕМАТИЧНА ОСВІТА

Видається з 2013.
ISSN 2413-158X (online)

ISSN 2413-1571 (print)

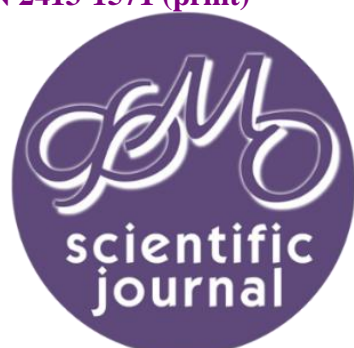

Шмельцер Неллі, Кляйне Міхаель. Базова освіта та основоположні ідеї - зрозуміле поєднання математичних структур. Фізико-математична освіта. 2020. Випуск 1(23). С. 177-185.

Schmelzer Nelli, Kleine Michael. Basic education and fundamental ideas - clear combination of mathematical structures. Physical and Mathematical Education. 2020. Issue 1(23). P. 177-185.

DOI 10.31110/2413-1571-2020-023-1-029

Nelli Schmelzer

Universität Bielefeld, Germany nelli.schmelzer@uni-bielefeld.de

Michael Kleine

Universität Bielefeld, Germany michael.kleine@uni-bielefeld.de

\title{
BASIC EDUCATION AND FUNDAMENTAL IDEAS - CLEAR COMBINATION OF MATHEMATICAL STRUCTURES
}

\section{ABSTRACT}

Formulation of the problem. At present, the focus on competence is an important part of discussions about mathematics lessons. In such discussions, particular attention is given to basic mathematical education. In this article, we substantiate the importance of mathematical competence in mathematical work and introduce mathematical work as a modeling cycle. The focus is on the processes of transforming reality into mathematics. In particular, transformational processes contribute to a better mathematical understanding of students and thus contribute to improving the quality of teaching of mathematics.

Materials and methods. In order to achieve our goals, we use in this article an empirical methods and general methods of scientific cognition: benchmarking to clarify different views on a problem and determining the direction of research, systematization and generalization to formulate conclusions and recommendations, summarize the author's pedagogical experience and observations.

Results. In Chapter 1.2, we describe the process of mathematical work at different stages, using the example of a typical problem. The above example is intended to clearly disclose the processes of thinking and work according to the theoretical model proposed in Chapter 1.1. It should also not be assumed that in a general situation, mathematical work can be comprehensively described by the example that we are studying. However, a competency-oriented teaching methodology is used to help students develop new strategies and heuristics to work with mathematics as a science. In order for students to develop their mathematical competence, mental models are called, which we call fundamental ideas. The construction of such cognitive structures is called the formation of fundamental ideas. This process is characterized by fixing the meaning of the new terms in terms of known factual connections, constructing mental objects that describe the term, and applying this objects to new contexts. Training involves both extending and changing existing foundational ideas as well as building new ideas. Accordingly, in Chapter 2.1 we use an example of probability to illustrate how various aspects of probability can be understood in terms of such a fundamental concept and how the development of fundamental ideas can occur. Significant in this article is a new approach that focuses on competence with a modeling cycle and a basic conception of foundational ideas.

Conclusions. The approach developed emphasizes the importance of considering mathematical work as a process and linking the individual levels of foundational ideas to a basic concept. The use of the proposed structure enables teachers to more effectively identify, interpret, and appropriately remove misunderstood students' basic mathematical ideas.

KEY WORDS: basic mathematical ideas, mathematical competence, modeling cycle, transformation of reality, mental objects, probability

\section{EINFÜHRUNG}

Die Kompetenzorientierung ist derzeit ein wesentlicher Bestandteil der Diskussionen um den Mathematikunterricht. Ein besonders wichtiger Fokus wird bei solcher Diskussionen auf das mathematische Grundbildung gelegt. In diesem Artikel konkretisieren wir die Bedeutung der mathematischer Kompetenz im Hinblick auf das mathematische Arbeiten und stellen das mathematische Arbeiten als Modellierungskreislauf vor. Dabei stehen die Übersetzungsprozesse zwischen der Realität und Mathematik im Mittelpunkt. Damit die Schüler und Schülerinnen ihre mathematische Kompetenz aufbauen können, sind die mentale Modelle erforderlich, welche von uns als Grundvorstellungen bezeichnet werden. Dementsprechend wird im zweiten Teil der Arbeit an dem Beispiel einer Wahrscheinlichkeit illustriert, wie verschiedene Aspekte einer Wahrscheinlichkeit mit einem solchen Grundvorstellungsbegriff zu verstehen sind und wie die Aufbau der Grundvorstellungen funktionieren kann. Dies geschieht mit der ausführlicher Auseinandersetzung mit dem Modellierungskreislauf nach Blum und Leiss (2005) und auch mit dem tieferen Einblick in den Grundvorstellungskonzept von Hofe (1995). Ein ausführlicher Beitrag zu diesem Thema findet sich auch bei Kleine (2012). 


\section{Mathematische Grundbildung.}

1.1. Begriffsfindung. Der Anspruch Schülerinnen und Schülern so in einem schulischen System zu unterrichten, dass sie in die Lage versetzt werden im täglichen Leben und in der Berufswelt ein mathematisches Fundament zu haben, auf das sie ihr Handeln und ihre Entscheidungen beziehen, ist vermutlich schon so alt, wie Mathematik als ein allgemeinbildendes Unterrichtsfach selbst. Die gegenwärtige Diskussion eines Konzepts «mathematischer Grundbildung» lenkt den Blickwinkel auf einen spezifischen Teil innerhalb dieses Konzepts, der als Kompetenzorientierung bezeichnet wird. Das hier zugrunde gelegte Kompetenzverständnis wurde dabei von Weinert geprägt.

«Dabei versteht man unter Kompetenz die bei Individuen verfügbaren oder durch sie erlernbaren kognitiven Fähigkeiten und Fertigkeiten, um bestimmte Probleme zu lösen, sowie die damit verbundenen motivationalen, volitionalen und sozialen Bereitschaften und Fähigkeiten um die Problemlösungen in variablen Situationen erfolgreich und verantwortungsvoll nutzen zu können.» (Weinert, 2001, S. 27-28)

Dieses Verständnis bedarf einer genaueren Klärung, wie es insbesondere für den Mathematikunterricht zugeschnitten werden kann. Grundsätzlich kann man aus der Definition von Weinert ein funktionales Verständnis des Kompetenzbegriffs herauslesen: die Bewältigung bestimmter Anforderungen wird als ein Indikator von Kompetenz betrachtet. Aufgrund von beobachtbaren Ergebnissen und Äußerungen in mathematischen Anforderungssituationen können wir demzufolge auf das Vorhandensein entsprechender mathematischer Kompetenzen schließen. Es reicht also nach diesem Verständnis nicht mehr aus bei Schülerinnen und Schülern ein nebulöses Begabungskonzept zu vermuten, dass im Moment nur nicht von dem Lernenden gezeigt wird. Nein, vielmehr wird nur dann von Kompetenz gesprochen, wenn die jeweilige Schülerin oder der jeweilige Schüler auch gewillt ist, sein Wissen in verschiedenen Situationen einzubringen und aktiv zu gebrauchen.

Der Definition wohnt auch ein bereichsspezifisches Verständnis inne: Kompetenzen werden dabei auf einen begrenzten Bereich von Kontexten und Situationen bezogen. Somit kann aufgrund der erfolgreichen Bewältigung entsprechender Sachzusammenhänge auf die Kompetenz geschlossen werden, die für die Bearbeitung dieser Inhalte notwendig ist. In der Mathematik lassen sich somit auch inhaltsbezogene Aussagen in verschiedenen Teilgebieten oder Teilaspekten unterscheiden. Wiederum sind die Kompetenzen natürlich auch nicht losgelöst voneinander, sondern Ausdruck eines allgemeinen Verständnisses. Demnach lassen sich Kompetenzen als Dispositionen auffassen, also etwas was tief in unseren kognitiven Strukturen verankert ist und somit über die Beschreibung einer einzelnen Leistung beispielsweise bei einer spezifischen Aufgabe allein hinausgeht.

Kompetenzen werden als prinzipiell erlernbar angesehen, worin sich eine Auffassung der Expertise-Forschung widerspiegelt, dass grundsätzlich jede Person Experte auf jedem Gebiet werden kann, wenn sie sich nur ausreichend mit einem Gegenstandsbereich beschäftigt. Vor der weiteren fachspezifischen Präzisierung steht im Folgenden zunächst einmal die Frage nach dem Verständnis schulischer Bildung, da gerade ja der Mathematik auch ein allgemeinbildender Status zuerkannt wird. Aus erziehungswissenschaftlicher Sicht ist eine allgemeine Bildung eine Voraussetzung des Individuums für die Teilnahme am gesellschaftlichen Leben. Diese Richtung findet sich in der Mathematikdidaktik auch bei Winter $(1976,1996)$ wieder, wenn er zur Allgemeinbildung all des Wissen, die Fertigkeiten, Fähigkeiten und Einstellungen zählt, die für einen Menschen in seinem gesellschaftlichen Umfeld notwendig erscheinen, unabhängig von seinem Geschlecht, Beruf, seiner Religionszugehörigkeit usw. Die ständigen Wandlungsprozesse und die immer schnelleren Umwälzungen in unserer Umwelt, seien sie von politischer, ökonomischer oder sozio-kultureller Art, machen es zwar auf der einen Seite im schwieriger einzugrenzen, was den Allgemeinbildung ist bzw. was Allgemeinbildung umfassen sollte. Auf der anderen Seite wird es aber immer wichtiger, dass möglichst viele Menschen eine möglichst fundierte und breite Bildung erwerben eben genau aufgrund dieser tiefgreifenden Wandlungsprozesse. Eine derartig verstandene Allgemeinbildung wird nach Winter im Mathematikunterricht dadurch vermittelt, dass den Schülerinnen und Schülern drei «Grunderfahrungen» ermöglicht werden, die sich als

(G1) Anwendungsorientierung,

(G2) Strukturorientierung und

(G3) Problemorientierung

charakterisieren lassen.

Die Anwendungsorientierung (G1) bedeutet in diesem Zusammenhang die Vermittlung grundlegender Einsichten in Natur, Gesellschaft und Kultur aus mathematischer Sicht. Die Strukturorientierung (G2) bezeichnet die Auseinandersetzung mit mathematischen Gegenständen und Sachverhalten in der Auffassung einer deduktiv geordneten Welt. Dahinter steckt ein strukturelles Wesen der Mathematik, die ausgehend von theoretischen Auffassungen und Grundsätzen eine Mathematik entstehen lässt, innerhalb derer sich mathematische Erkenntnisse sachlogisch ableiten lassen. Die Anwendung auf eine äußere Welt außerhalb der Mathematik ist hierbei dann zweitrangig. Demgegenüber betont die Problemorientierung (G3) genau den umgekehrten Fall, nämlich dass es auch zu einem Wesen der Mathematik gehört heuristische Fähigkeiten zu erwerben, um in Problemlöseprozessen Muster zu erkennen, diese zu beschreiben und zu nutzen. Alle drei Grunderfahrungen sollen eng miteinander verbunden sein. Eine einseitige Betonung - egal in welche Richtung - ist für ein Verständnis allgemeiner mathematischer Bildung somit kontraproduktiv.

Mathematischer Schulunterricht soll die Voraussetzungen schaffen, diese grundlegenden mathematischen Kenntnisse zu vermitteln, um in unterschiedlichen Kontexten Einsichten auf reflektierende und einsichtige Weise zu gewinnen. Diese Sichtweise setzt die Ideen des Mathematikers und Fachdidaktikers Freudenthal fort, wonach die Vermittlung von Zusammenhängen ein wesentliches Ziel mathematischer Unterweisung ist. Aus den bisherigen Überlegungen lässt sich nun ein Verständnis für mathematische Kompetenz als ein individuelles Merkmal entwickeln, das im Weiteren den Ausführungen zugrunde gelegt wird. Demnach umfasst mathematische Kompetenz nicht nur die Beherrschung mathematischer Sätze, Regeln und Verfahren (aber auch!), sondern sie zeigt vor allem sich in einem verständnisvollem Umgang der Mathematik indem mathematische Kenntnisse in einer Vielzahl von Kontexten bewusst und reflektiert eingesetzt werden können.

Diese weitreichende Bedeutung mathematischer Kompetenz ist sicherlich schwer in einem Unterricht umzusetzen und zu überprüfen. Es besteht also nun die Aufgabe in der Konkretisierung einer solchen Sichtweise im Hinblick auf mathematisches Arbeiten, wie es in der Schule umgesetzt werden kann. 
1.2. Mathematisches Arbeiten als Prozess. In der zuvor beschriebenen Auffassung zeichnet sich mathematische Grundbildung unter anderem durch eine Anwendungsorientierung aus. Dieses Verständnis soll als Ausgangspunkt der folgenden Ausführungen dienen, um daran die anderen Aspekte sukzessive zu ergänzen. Im folgenden Verständnis wird mathematisches Arbeiten über die Auseinandersetzung mit realen Problemen gelehrt, um erworbene Kenntnisse später in realen Kontexten anwenden zu können. Im Sinne eines induktiven Vorgehens ist es dabei günstig, wenn die zu bildenden mathematischen Begriffe von Schülerinnen und Schülern möglichst selbst aus Umweltbezügen herausgelöst werden, damit sie diese auch später wieder auf solche Bezüge anwenden können. Mathematisches Arbeiten durch die Herauslösung von Umweltbezügen wird in der fachdidaktischen Literatur oftmals als Prozess des Modellierens bezeichnet, wie er im Folgenden in einer Auffassung von Blum und Leiss (2006) dargestellt wird.

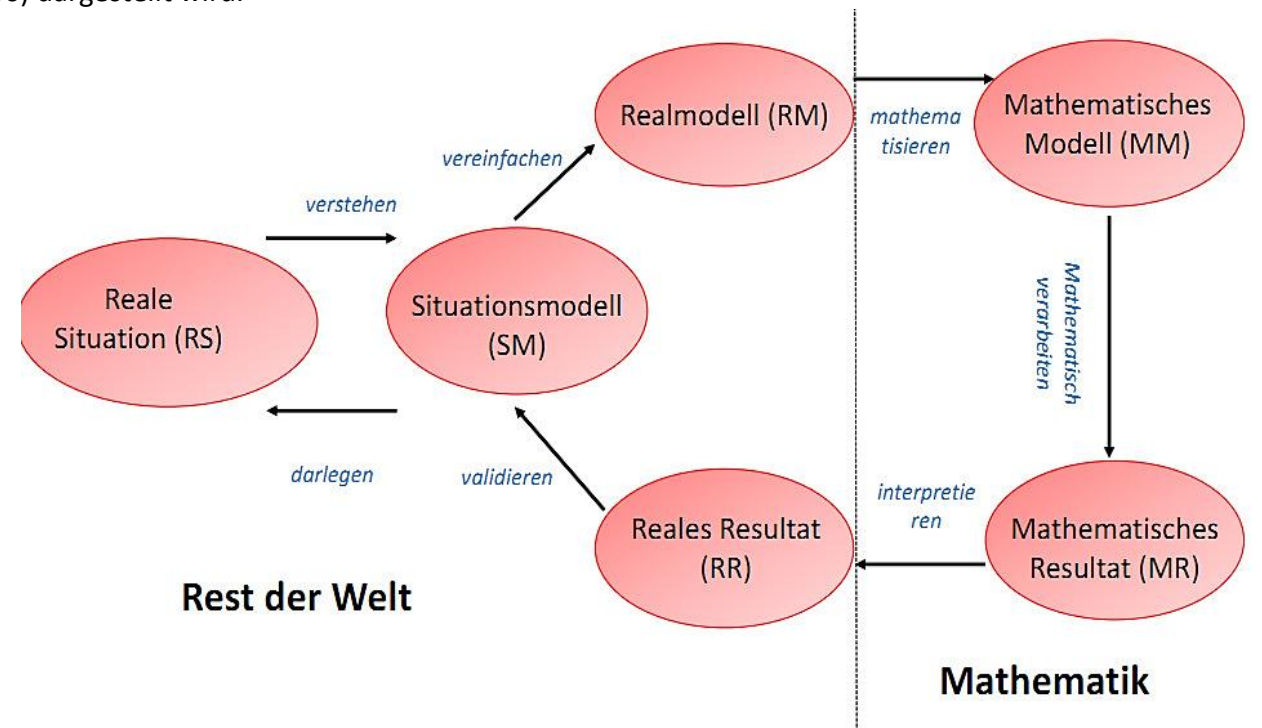

Abbildung 1. Mathematisches Arbeiten als Prozess nach Blum und Leiss (2005)

In Abbildung 1 sind kognitive bzw. reale Phasen eines Modellierungsprozesses als Ellipsen dargestellt. Ein solcher Prozess hat theoretisch den folgenden prototypischen Ablauf: Ausgangspunkt (Phase 1) ist eine reale Situation (RS) mit einer Fragestellung, die entweder selbst gestellt oder vorgegeben wird. Dabei ist die reale Situation durch ihren Kontext gekennzeichnet, die Art der Präsentation, Aspekte wie Diagramme, Bilder oder ähnliches, durch das die Situation dargeboten wird. Im Kopf einer jeden Person setzen nun Denkprozesse ein um diese Situation zu verstehen und einzuordnen. Dabei spielen Erfahrungen innerhalb des Kontextes ebenso eine Rolle wie die Tatsache, auf welche Weise Begrifflichkeiten semantisch erfasst, Schaubilder eingeordnet und verstanden werden. Der Prozess wird als «verstehen» bezeichnet und es bildet sich ein individuelles Situationsmodell (SM), das für jede Person anders aussehen kann. Im Hinblick auf eine Aufgabe oder Fragestellung werden nun - abhängig von der Komplexität einer Situation Vereinfachungen vorgenommen (Phase 2), also im Grunde die Art von Idealisierungen, die zu mathematischen Begriffen führen. Dazu zählen auch alle Arten von Annahmen, die getroffen werden und die dem weiteren mathematischen Prozess zugrunde gelegt werden. Aus den Vereinfachungen entsteht ein Realmodell (RM), das im Gegensatz zur realen Situation schon «mathematisch zugeschnitten» wurde. Dieses Realmodell muss nun (Phase 3) weiter strukturiert werden, indem den mathematischen Begriffen und Annahmen jetzt mathematische Inhalte zugeordnet werden, also das Modell mathematisiert wird.

Die Mathematisierung kann sowohl in der konkreten Auswahl mathematischer Formeln bestehen, die Berechnungszusammenhänge zwischen beteiligten Größen herstellen, als auch in der grundsätzlichen Entscheidung für einen mathematischen Zusammenhang, etwa wenn man der Einfachheit halber einen linearen und keinen exponentiellen Zusammenhang zwischen Größen zugrunde legt. Man erhält am Ende des Mathematisierungsvorgangs ein mathematisches Modell (MM), in dem sämtliche Zusammenhänge vorliegen, mit denen im Weiteren gearbeitet wird. Auf der Grundlage dieses mathematischen Modells beginnt nun (Phase 4) das mathematische Verarbeiten. Hierbei kann es sich sowohl um einen rechnerischen Vorgang, aber auch um ein argumentatives oder zeichnerisches Vorgehen handeln. Ziel dieser Phase ist es ein mathematisches Resultat (MR) zu erhalten.

Im weiteren Schritt (Phase 5) muss das mathematische Resultat interpretiert werden. Typischerweise fragt man sich hier nach der Existenz mathematischer Lösungen in der Realität. Die Interpretation mündet in einem realen Resultat (RR), das in einem unmittelbaren Bedeutungszusammenhang in der Umwelt steht. Ob dieser Bedeutungszusammenhang jedoch auch einen Bezug zur Ausgangssituation hat, muss im Weiteren (Phase 6) geklärt werden. Dazu wird das reale Resultat validiert. Hierzu können Indikatoren herangezogen werden, die sich aus Vermessungen, Datenbeständen oder Recherche in weiteren Kontexten ergeben. Aber auch die Prüfung der Annahmen gehört dazu. War die Vereinfachung möglicherweise in einigen Bereichen zu stark verzerrend? War der mathematische Ansatz angemessen? Die Validierung erfolgt dabei am Situationsmodell (SM), denn das war ja der Ansatz, der das Verständnis des Individuums von der Ausgangssituation beschreibt. Im Zweifelsfall führt der Prozess wieder zu einem neuen Realmodell (Phase 7), indem die Vereinfachungen neu gestaltet und vorgenommen werden in der Hoffnung ein neues Resultat zu erhalten, das der Validierung besser stand hält. Im Falle einer vorläufigen Zufriedenheit mit dem realen Resultat wird das Ergebnis ausgehend vom Verständnis aus dem Situationsmodell nun dargelegt und wieder auf die reale Situation (RS) bezogen. Der Prozess wird somit durch ein kommunizierbares Ergebnis abgeschlossen.

Innerhalb des Prozesses wird deutlich zwischen zwei Ebenen unterschieden, in denen man sich in dem Arbeitsablauf bewegt: der mathematischen Ebene auf der einen Seite, die sich an der mathematischen Struktur orientiert und hinter dem sich 
insbesondere das deduktive Verständnis einer mathematischen Grundbildung im zuvor dargelegten Sinne zeigen kann. Auf der anderen Seite steht der "Rest der Welt", der in seinen Wesenszügen einen induktiven Zugang zur Mathematik nahelegt. Beide sind miteinander verbunden. Bevor wir im folgenden Abschnitt jedoch diese Nahtstelle genauer ins Auge fassen wollen, sollen die theoretischen Ausführungen zunächst einmal an einem Beispiel ausführlich illustriert werden.

\section{Aufgabe 1.1. Zeitungsartikel.}

\section{Bombenfund: Evakuierung in Vechta}

Vechta. Eine Bombe aus dem Zweiten Weltkrieg ist am Mittwoch in der Stadt Vechta gefunden worden. Der Blindgänger sollte noch am Nachmittag entschärft werden, teilte die Stadtverwaltung mit. Alle Anwohner in einem Radius von 1.000 Metern rund um den Fundort wurden aufgefordert, das Gebiet zu verlassen. Von der Evakuierung waren auch eine Schule und zwei Kindergärten betroffen. In einem nahe gelegenen Schulzentrum wurde für die Anwohner ein Evakuierungszentrum eingerichtet.

aus: Hamburger Abendblatt vom 31.08.2011
Oftmals werden auch Turnhallen als Notunterkünfte vorgesehen, in denen Menschen auch mehrere Tage zubringen müssen. Wie viele Menschen können in der Turnhalle deiner Schule zum Übernachten als Notunterkunft untergebracht werden? Stelle deine Überlegungen übersichtlich dar.

Für diese Aufgabe kann man den Prozess des mathematischen Arbeitens wie folgt beschreiben:

(Phase 1) Ausgehend von der gegebenen realen Situation aus dem Zeitungsausschnitt geht es darum zu verstehen, was das inhaltliche Anliegen dieser Aufgabe ist. Die Verstehensprozesse finden nun auf unterschiedlicher Ebene statt: sprachliche Hürden können je nach Altersstufe in der semantischen Bedeutung von Begriffen wie «Blindgänger» oder «Evakuierung» stecken. Diese sprachlichen Barrieren werden jedoch durch die Vorsätze deutlich reduziert. Ebenso gehören Assoziationen zu diesem Schritt, die davon abhängen, ob Schülerinnen und Schüler schon einmal ein Bild von einem Bombenfund im Fernsehen oder Zeitungen gesehen habe. Beispielsweise kann man eine verschlammte und verrostete Rohrbombe vor Augen haben, die man manchmal in Zeitungen sieht. Solche Assoziationen können natürlich durch ein Bild gelenkt werden. Zum Verstehensprozess kommt die Intention der Aufgabe hinzu: Es soll um eine Notunterkunft in einer Turnhalle gehen, die auch zum Schlafen genutzt werden kann. Der Fokus wird dabei auf die eigene Schulturnhalle gehängt, so dass ein Bild vom Zustand und dem Aussehen der eigenen Schulturnhalle vor dem Auge vorhanden sein wird. Und es scheint bei dieser Aufgabe um eine Kapazitätsgrenze zu gehen, also einer Obergrenze, wie viele Personen maximal in einer Turnhalle untergebracht werden können. Am Ende dieses Schrittes hat sich dann beispielsweise das dargelegte Verständnis als Situationsmodell ausgebildet, das individuell geprägt ist.

(Phase 2) Von diesem Situationsmodell aus werden nun Vereinfachungen getroffen in Form von Annahmen und Idealisierungen. Eine Idealisierung besteht nun darin die Turnhalle für die weiteren Betrachtungen als Quader anzusehen bzw. für Schlafplätze nur noch den Boden zu betrachten und dabei von einem Rechteck auszugehen. Befestige Gerätschaften an den Wänden, Ausbuchtungen, Verkleidungen usw. werden außer acht gelassen. Man kann dann annehmen, dass jeder Person, unabhängig von Größe und Alter, als Schlafplatz eine Matratze zur Verfügung gestellt wird, die der Einfachheit halber $2 \mathrm{~m}$ lang und $1 \mathrm{~m}$ breit ist und somit eine Fläche auf dem Boden von $2 \mathrm{~m}^{2}$ einnimmt. Ebenso werden keine Einschränkungen hinsichtlich von Schlafbereichen bezüglich des Geschlechts gemacht. Die Matratzen dürfen in einer Reihe liegen, zwischen den Reihen muss jedoch ein Gang von $1 \mathrm{~m}$ Breite bleiben. Die Maße des Turnhalleninnenraums werden abgemessen, beispielsweise $24 m \times 33 m$. Somit stellt dieses reale Modell eine Verengung der Ausgangssituation dar.

(Phase 3) Das reale Modell muss nun mathematisiert werden (die Mathematisierung der Matratzenfläche kann natürlich auch hier erfolgen, da sollte man aber auch keine zu puristische Auffassung vertreten). Es gibt jetzt verschiedene Möglichkeiten, wie in der Abbildung 2 zu sehen ist.

(Phase 4) Entsprechend des gewählten Vorgehens können, wie in der Abbildung 3 dargestellte unterschiedliche mathematische Verarbeitungen sich ergeben.

(Phase 5) Die Bedeutung des mathematischen Ergebnisses ist in der Realität jeweils die Anzahl an Matratzen, die maximal bei den gegebenen Bedingungen in eine Turnhalle passen und damit dann auch die Anzahl an Personen. Folglich passen maximal 264 Personen zum Übernachten in die Unterkunft.

(Phase 6) Eine Validierung des Ergebnisses ergibt insbesondere bei der graphischen Lösung augenscheinlich ein Problem: Die Matratzen liegen zu eng, das es keinen freien Zugang zu den einzelnen Reihen gibt etwa von einer Turnhallentür aus. Sicherlich wird neben dem reinen Schlafplatz auch etwas Platz für persönliche Gegenstände benötigt. Bei der Validierung kann man auch Vorschriften erkunden lassen etwa die Brandschutzverordnung, nach der Rettungswege mit einer Breite von mindestens 1,20m freigehalten werden müssen. Ebenso hängt die Anzahl der Personen von den hygienischen Bedingungen ab, die unter anderem durch die Anzahl von Toiletten und Waschgelegenheiten gegeben ist. Solche Überarbeitungen der Annahmen können dann zu einem neuen Realmodell führen.

\begin{tabular}{|l|l|l|}
\hline \multicolumn{1}{|c|}{ rechnerisch: } & \multicolumn{1}{c|}{ graphisch: } & \multicolumn{2}{c|}{ argumentativ: } \\
\hline $\begin{array}{l}\text { Idee: Wie oft passt die } \\
\text { Matratzenfläche mit Gang } \\
\text { in die Hallenfläche? }\end{array}$ & $\begin{array}{l}\text { Idee: Parkettierung der } \\
\text { Hallenfläche mit Matratzen und } \\
\text { Gang. }\end{array}$ & $\begin{array}{l}\text { Idee: Flächenauslegung bei } \\
\text { Rechtecken auf die Situation } \\
\text { übertragen. }\end{array}$ \\
$\begin{array}{l}\text { A Halle }=\mathrm{a}^{*} \mathrm{~b} \\
\text { Aschlaffflache }=2 \mathrm{~m}^{2}+1 \mathrm{~m}^{2}\end{array}$ & &
\end{tabular}

Abbildung 2: Verschiedene Möglichkeiten, Phase 3 


\begin{tabular}{|c|c|c|}
\hline rechnerisch: & graphisch: & argumentativ: \\
\hline $\begin{array}{l}A=24 m * 33 m=792 m^{2} \\
792 m^{2}: 3 m^{2}=264\end{array}$ & 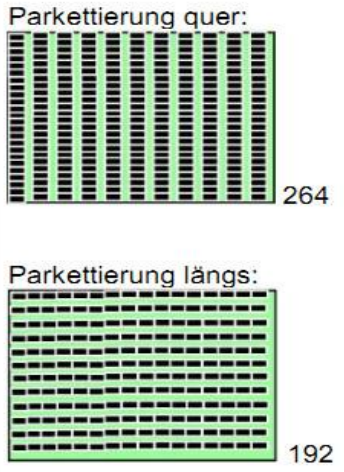 & $\begin{array}{l}\text { In eine Reihe passen } 24 \\
\text { Matratzen. Untereinander lassen } \\
\text { sich } 11 \text { Reihen legen, also } \\
\text { insgesamt } \\
\qquad 24 \text { * } 11=264\end{array}$ \\
\hline
\end{tabular}

Abbildung 3. Unterschiedliche mathematische Verarbeitungen, Phase 4

(Phase 7) Man kann sich natürlich auch bei Kenntnis der Unzulänglichkeiten einer Obergrenze eigenen Rechenweges, mit der Bestimmung zufrieden geben. Das Ergebnis müsste dann dargelegt werden, sowohl hinsichtlich der Annahmen, des gewählten Bearbeitungsweges, als auch der Verbesserungsmöglichkeiten des Modells. Dieses würde dann für diese reale Situation die Aufgabe abschließen.

Betrachtet man die Anforderungen in dieser Aufgabe vom mathematischen Gehalt her, dann werden dort typische geometrische Inhalte zu Beginn der Sekundarstufe I angesprochen.

Das vorgestellte Beispiel soll explizit die Denk- und Arbeitsprozesse entsprechend des theoretischen Modells offen legen. Es soll auch nicht der Eindruck entstehen, dass mathematisches Arbeiten nur in diesem Umfang, mit dieser Offenheit und in dieser Strukturierung als kompetenzorientierter Mathematikunterricht zu verstehen ist. Es stellt jedoch eine Zielmarke des Mathematikunterrichts dar, dass Schülerinnen und Schüler Mathematik auch von dieser Seite kennen lernen und Strategien und Heuristiken entwickeln, um mit ihnen umzugehen.

Im folgenden Abschnitt soll der Übergangsprozess zwischen der mathematischen und der realitätsbezogenen Ebene in den Blick genommen werden, weil diese Nahtstelle eine wesentliche Rolle für die Beschreibung mathematischer Grundbildung spielt.

\section{Grundvorstellungen als Vermittler zwischen Realität und Mathematik.}

Im dargelegten Verständnis mathematischen Arbeitens stellen die Übersetzungen zwischen Realität und Mathematik zentrale Tätigkeiten dar; hierbei wird einerseits ein reales Modell mathematisiert und andererseits das mathematische Ergebnis in Bezug auf die realen Konsequenzen interpretiert. Für diese Übersetzungsprozesse sind nach Freudenthal (1983) tragfähige mentale Objekte mathematischer Begriffe für das Verständnis erforderlich, die der Begriffsbildung vorausgehen. In der deutschen Mathematikdidaktik bezeichnet man solche kognitiven Objekte traditionell als Grundvorstellungen (vgl. vom Hofe, 1995). Als Kernpunkte dieses Konzepts stellt vom Hofe (2003) drei wesentliche Merkmale heraus:

1) Es gibt keine eindeutige Zuordnung zwischen mathematischen Objekten und spezifischen Grundvorstellungen, weil in der Regel mathematische Inhalte durch mehrere Grundvorstellungen erfasst werden können, die in Beziehung zueinander stehen.

2) Man kann zwischen zwei Arten von Grundvorstellungen unterscheiden: Auf der einen Seite existieren primäre Grundvorstellungen, deren Wurzeln auch in der Zeit vor einen Mathematikunterricht liegen können und die sich durch gegenständliche Handlungen und konkrete Operationen auszeichnen. Auf der anderen Seite werden während einer fortlaufenden Mathematikunterricht sekundäre Grundvorstellungen aufgebaut, die vor allem durch mathematische Darstellungsbezüge gekennzeichnet sind.

3) Grundvorstellungen sind nicht statisch und universell valide, sondern dynamisch und entwickeln sich innerhalb eines vernetzten Systems weiter. Die Notwendigkeit für Entwicklungen resultiert aus ihrem weitgehend bereichsspezifischen Geltungsbereich: Erweisen sich Grundvorstellungen innerhalb eines mathematischen Inhaltsbereichs als tragfähig, so müssen diese möglicherweise in anderen Bereichen erweitert werden.

Der Aufbau solcher kognitiven Strukturen wird als Ausbilden von Grundvorstellungen bezeichnet. Dieser Prozess ist gekennzeichnet durch die Erfassung der inhaltlichen Bedeutung neuer Begriffe über bekannte Sachzusammenhänge, durch den Aufbau mentaler Objekte, die den Begriff repräsentieren sowie durch die Anwendung auf neue Kontexte. Die Ausbildung umfasst dabei sowohl die Erweiterung und Veränderung vorhandener Grundvorstellungen als auch den Aufbau neuer Vorstellungen. Grundvorstellungen lassen sich vor diesem Hintergrund kennzeichnen als spezifische (a) mentale Objekte, die insbesondere strukturelle und funktionale Aspekte eines mathematischen Gegenstandes abbilden; als (b) dynamische Objekte, die sich im Laufe der Zeit durch neue Erfahrungen verändern und entwickeln können und als (c) Elemente eines kognitiven Netzes, in dem einzelne Vorstellungen nicht isoliert sind, sondern in Wechselbeziehungen zu anderen Vorstellungen stehen. Diese Charakterisierungen weisen bereits darauf hin, dass sich Grundvorstellungen der unmittelbaren Observation entziehen und der Validierung durch beobachtbares Verhalten bedürfen. Diese didaktische Sichtweise stellt den deskriptiven Aspekt des Konstrukts heraus: Durch eine Analyse des individuellen Verhaltens (etwa in Unterrichtssituationen, Interviews, Tests) wird versucht die jeweiligen Vorstellungen von mathematischen Begriffen zu rekonstruieren. Dieser Betrachtung steht der normative Aspekt gegenüber, wonach Grundvorstellungen als Leitlinien für den Aufbau mentaler Repräsentationen mathematischer Inhalte verwendet werden. Während also beim ersten Aspekt das handlungsleitende Interesse in der Frage steckt, welche Vorstellungen Schülerinnen und Schüler tatsächlich aktiviert haben, liegt es im zweiten Aspekt in der Frage, welche Vorstellungen Schülerinnen 
und Schüler ausbilden sollen. Vergleicht man die vorhandenen mit den erwünschten Vorstellungen, so kommt es im Idealfall zu einer Übereinstimmung. Oftmals werden jedoch Defizite sichtbar, deren Feststellung und Analyse ein zentrales Thema der Fachdidaktik in der Auseinandersetzung mit Grundvorstellungen ist.

Also teilt man die Grundvorstellungen in drei Dimensionen mit deren verschiedene didaktischen Sichtweisen aus der Lehrerperspektive, Schülerperspektive und Fachanforderungen auf. In der Abbildung 4 sind die Grundvorstellungsdimensionen mit den charakteristischen Leitfragen zu jeweiliger Dimension dargestellt.

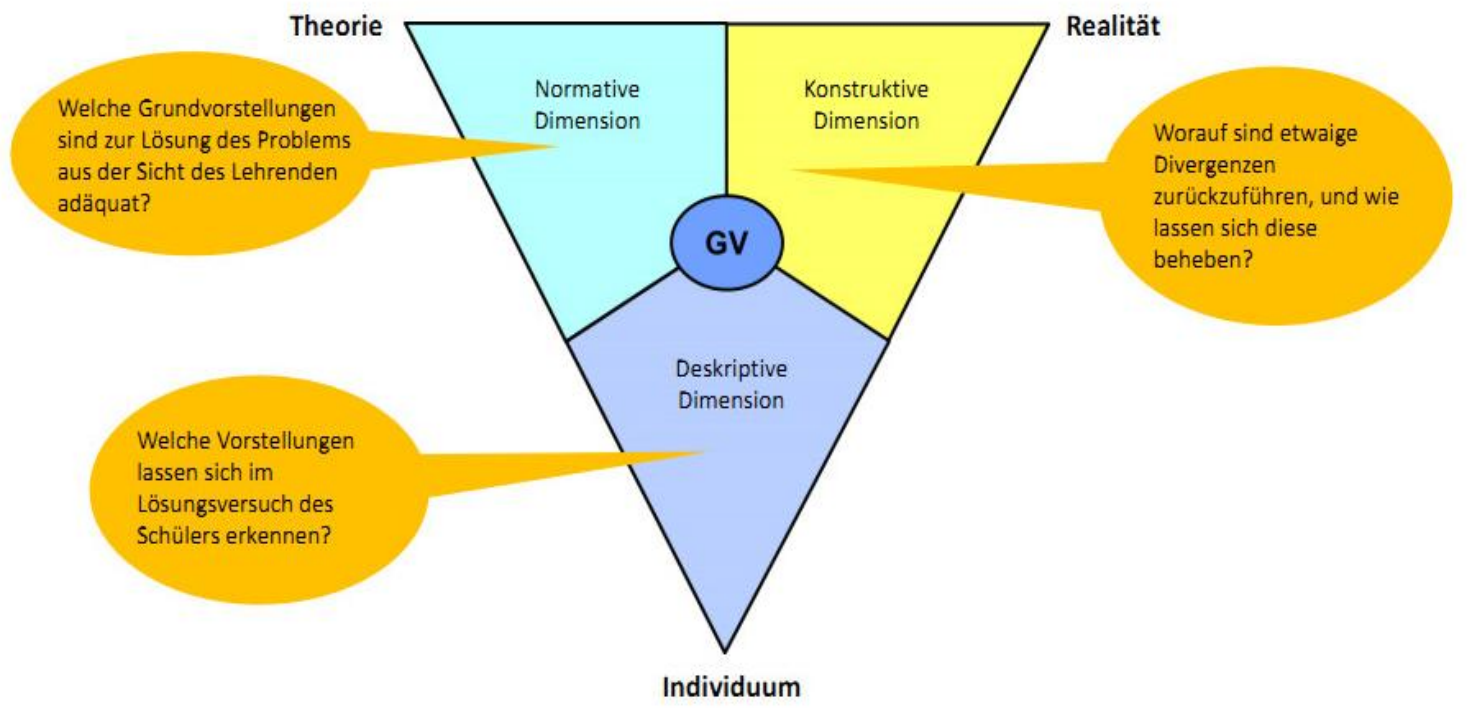

Abbildung 4. Grundvorstellungsdimensionen mit Leitfragen

Für die Anwendung dieses theoretischen Arbeitsmodells ist es empfehlenswert mit der normativer Dimension anzufangen, hier werden die geforderte mathematische Inhalte aus der Sicht des Experten analysiert und die tragfähige Grundvorstellungen ausgearbeitet. Danach werden unterschiedliche Methoden zur Förderung der Aufbau der Grundvorstellungen ausgearbeitet und in das Unterricht implementiert. In der nächsten Phase werden die Schüler und Schülerinnen beobachtet und deren Lösungsansätze samt deren Vorstellungen zu den mathematischen Inhalten analysiert und interpretiert. Letztendlich stellt man fest, dass die Lernenden geeignete Vorstellungen auch teilweise richtige Vorstellungen oder sogar Fehlvorstellungen entwickeln. In den Fällen, wo Aufbau der Grundvorstellungen nicht oder nur teilweise funktioniert hat, muss die Lehrkraft in der ersten Linie herausfinden, warum es nicht funktioniert hat und danach Handelsalternativen suchen.

So ein Modell zu betrachten erweist sich als sinnvoll, da es einerseits dem Prinzip kumulativen Lernens entgegen kommt, andererseits auch für die Gestaltung von Lern- und Diagnoseaufgaben eine sinnvolle Orientierungshilfe bietet. Im Folgenden werden exemplarisch die Grundvorstellungen zum Wahrscheinlichkeitsbegriff aus der Perspektiven der drei Grundvorstellungsdimensionen dargestellt und analysiert.

2.1. Grundvorstellungen zum Wahrscheinlichkeitsbegriff. Um über die Grundvorstellungen des Wahrscheinlichkeitsbegriff sprechen zu können, werden im Folgenden als erstes die theoretischen Grundlagen zum Wahrscheinlichkeitsbegriff geschaffen.

Wir definieren eine Wahrscheinlichkeit als ein Maß für das Eintreten eines Ereignisses in einem Zufallsexperiment. Bei einem Zufallsexperiment wissen wir, welche möglichen Ereignisse eintreten können, aber wir wissen noch nicht, welches Ereignis im konkreten Fall tatsächlich auftritt. Mit Wahrscheinlichkeit wird jedem Ereignis eine Art Gewicht gegeben um die Zuversicht über dieses Ereignis zu quantifizieren. Damit stellt sich die Frage, wie man zu Quantifizierung der Wahrscheinlichkeit gelangt.

Gegenwärtig findet man in der Literatur zum Wahrscheinlichkeitsbegriff zwei unterschiedliche Sichtweisen für eine Wahrscheinlichkeit. Die subjektivistische Wahrscheinlichkeit bezieht sich auf die Information, die ein Subjekt oder eine Gemeinschaft von dem betreffenden stochastischen System hat, also basiert sich auf Vermutungen und innere Überzeugung. Der subjektivistische Aspekt wird mit dem prognostischen Wahrscheinlichkeitsbegriff definiert.

Objektivistische Sichtweisen sind dagegen eher unabhängig von menschlichen Kenntnis und haben einen innermathematischen Charakter, man kann sie auch als intersubjektiv bezeichnen. Der objektivistischen Sichtweise werden unterschiedliche Definitionen des Wahrscheinlichkeitsbegriff zugeordnet: axiomatische (formale), klassische und frequentistische.

Die formale inhaltliche Deutung eines Wahrscheinlichkeitsbegriff basiert auf dem Axiomensystem von Kolmogorov, welches mathematische Eigenschaften von Wahrscheinlichkeiten festlegt. Diese Definition ist für den schulischen Mathematikunterricht nur in der Oberstufe denkbar, da die inhaltliche Interpretation der Wahrscheinlichkeit aus der Axiomen nicht gegeben ist. Demzufolge ist der Aufbau der Grundvorstellungen zum Wahrscheinlichkeitsbegriff durch den axiomatischen Zugang alleine, nicht geeignet.

Der frequentistische Wahrscheinlichkeitsbegriff beschreibt die Wahrscheinlichkeiten als Grenzwert der relativen Häufigkeiten in unendlich langen Versuchsserien. Wenn man ein Experiment beliebig oft wiederholen kann, kann man die relative Häufigkeit berechnen, indem man die Anzahl der Treffer durch die Anzahl der gesamten Versuche teilt. Der Grenzwert dieses Bruchs ist die gesuchte Wahrscheinlichkeit. Hierbei spielt das Gesetz der großen Zahlen eine zentrale Rolle. Voraussetzung hierfür ist die beliebige Wiederholbarkeit des Experiments. Außerdem müssen die einzelnen Durchgänge voneinander unabhängig sein. 
a) Der klassische Wahrscheinlichkeitsbegriff geht auf Laplace zurück und definiert die Wahrscheinlichkeit eines zufälligen Ereignisses als Anteil der zu diesem Ereignis günstigen Fälle gegenüber der Anzahl aller gleichmöglicher Fälle. Diese Definition der Wahrscheinlichkeit setzt voraus, dass alle elementaren Ergebnisse die gleiche Wahrscheinlichkeit haben.

b) Der prognostische Wahrscheinlichkeitsbegriff ist nicht im Rahmen innermathematischer Systematik erfahrbar, er hängt von derzeit verfügbarem Informationsstand ab. Bei manchen Zufallsereignissen kann deren Eintrittswahrscheinlichkeit nur geschätzt und nicht berechnet werden. Die Schätzung basiert in der Regel auf Expertenwissen, Erfahrung und Intuition. Diese Wahrscheinlichkeit kann man auch als Grad persönlicher Überzeugung auffassen.

Zu den letzten drei aufgezählten (b-d) Auffassungen des Wahrschenlichkeitsbegriffs definieren Malle und Malle (2003) folgende normative Grundvorstellungen:

GV1: Wahrscheinlichkeit als Maß für eine Erwartung. (Eine Wahrscheinlichkeit ist ein Maß für eine Erwartung. Der Grad der Erwartung wird durch eine Zahl von 0 bis 1 ausgedrückt)

GV2: Wahrscheinlichkeit als subjektives Vertrauen. (Als Wahrscheinlichkeit eines Ereignisses kann der Grad des subjektiven Vertrauens in das Eintreten des Ereignisses genommen werden)

GV3: Wahrscheinlichkeit als relative Häufigkeit. (Als Wahrscheinlichkeit eines Ereignisses kann die relative Häufigkeit des Eintretens des Ereignisses in einer Versuchsserie genommen werden)

GV4: Wahrscheinlichkeit als relativer Anteil. (Als Wahrscheinlichkeit eines Ereignisses kann der relative Anteil der zum Ereignis gehörenden Versuchsausfälle an allen möglichen Versuchsausfällen genommen werden)

Hier wird die Zuordnung der Grundvorstellungen zu den jeweiligen Wahrscheinlichkeitsbegriffen deutlich erkennbar. Die GV1 kann man zu allen drei Auffassungen zuordnen. Die GV2 beschreibt den prognostischen Wahrscheinlichkeitsbegriff. Die GV3 zeichnet den frequentistischen und die GV4 klassischen Wahrscheinlichkeitsbegriff aus.

Nach der Entstehung der Grundvorstellungstheorie in den neunziger Jahren, gab es in der Mathematikdidaktik verschiedene Forschungsrichtungen zu dieser. Einige Autoren versuchten die normative Grundvorstellungen zu formulieren, die Anderen haben deskriptive Ebene untersucht. Im Laufe der Zeit wurden Grundvorstellungen formuliert, die sehr schwer deskriptiv nachweislich sind.

Die normative Grundvorstellungsformulierung von Malle und Malle (2003) erlaubt es kaum diese direkt deskriptiv nachzuweisen, dementsprechend sind noch weitere normative Aspekte erforderlich um die deskriptive Verbindung zu den einzelnen Sachverhalten für die Wahrscheinlichkeit notwendig.

Im folgenden Abschnitt werden die theoretischen Ausführungen exemplarisch an zwei Aufgaben dargestellt:

Aufgabe 2.1. Münzenwurf 1. Welche der folgenden Möglichkeiten hältst du beim sechsmaligen Werfen einer Münze für am wahrscheinlichsten? K steht hierbei für das oben Aufliegen von Kopf und Z für das oben Aufliegen von Zahl.

○ $K Z K Z K Z$

○ $K K K K Z K$

- $K K K K K K$

- Alle oben angegebene Möglichkeiten haben gleiche Wahrscheinlichkeit.

Aufgabe 2.2. Münzenwurf 2. Wenn du eine Münze sechs Mal hintereinander wirfst und die Folge Z Z Z Z Z Z beobachtest. Was würdest du beim nächsten Wurf erwarten?

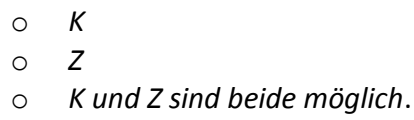

2.1.1. Normative Grundvorstellungen. Für diese Aufgaben kann man die normative Dimension der Grundvorstellungsebene, wie folgt beschreiben: Betrachtet man die Aufgabe mit der «Grundvorstellungsbrille» von Malle und Malle (2003), so ist es notwendig für die Bearbeitung und richtige Lösung der Aufgaben die Aufbau von GV1, GV4. Viel genauer muss es einerseits verstanden werden was die Gleichwahrscheinlichkeit in der klassischen Laplace Definition bedeutet, andererseits muss erkannt werden, dass jeder Münzenwurf von dem vorherigen stochastisch unabhängig ist.

Vorstellung1: Eine Münze hat 2 Seiten: Kopf und Zahl. Sofern bei der Münze nichts manipuliert wurde ist die Wahrscheinlichkeit Zahl zu werfen genau so groß, wie die Wahrscheinlichkeit Kopf zu werfen, jeweils $P(K)=P(Z)=0,5$. Dementsprechend ist die Anzahl möglichen Fälle beim Werfen einer Münze $W=\{K ; Z\}$ mit $n=2$ gleichwahrscheinlichen Elementarereignissen.

Theorem 2.3. Die Wahrscheinlichkeit eines Ereignisses $E$ aus dem Ereignisraum $W$ gleichwahrscheinlicher Elementarereignisse ist gegeben durch

$$
P(E)=\frac{g}{n}=\frac{\text { Anzahl günstiger Fälle }}{\text { Anzahl aller möglichen Fälle }}
$$

Definition 2.4. Von gleichwahrscheinlichen Elementarereignissen spricht man, wenn

$P\left(\left\{\omega_{1}\right\}\right)=P\left(\left\{\omega_{2}\right\}\right)=\ldots=P\left(\left\{\omega_{n}\right\}\right)=\frac{1}{n}$

mit $|W|=n$ und $\omega_{1}, \omega_{2}, \ldots, \omega_{n} \in W$.

Vorstellung 2: Wenn eine Münze mehrere Male hintereinander geworfen wird, ist jeder Wurf von dem Anderen stochastisch unabhängig. Die Wahrscheinlichkeit eine Zahl oder Kopf zu werfen bleibt 0,5 und zwar bei jedem Wurf, unabhängig davon, was bei früheren oder späteren Würfen auftritt.

Definition 2.5. Zwei Ereignisse sind stochastisch unabhängig falls gilt

$P(A \mid B)=P(A)$ oder $P(B \mid A)=P(B)$,

wobei $P(A \mid B)$ ist die bedingte Wahrscheinlichkeit von $A$ unter $B$ und $P(B \mid A)$ die bedingte Wahrscheinlichkeit von $B$ unter $A$. Umgeformt $P(A \cap B)=P(A) \cdot P(B)$. 
Somit ergeben sich die Wahrscheinlichkeiten in der ersten Aufgabe für alle drei Antwortmöglichkeiten $P(K Z K Z K Z)=P(K K K K Z K)=P(K K K K K K)=0,5^{6}$, so dass die letzte Antwort korrekt ist.

2.1.2. Deskriptive Grundvorstellungen. Zur deskriptiver Dimension der Grundvorstellungsebene sind im Folgenden Beispiele aus einer Untersuchung im Zusammenhang mit einer Bachelorarbeit dargestellt. Getestet wurden die Lehramtsstudierende mit Hauptfach Mathematik und ohne Haupt- oder Nebenfach Mathematik. Die Stichprobengröße ist sehr gering um die Verallgemeinerungen zu treffen, trotzdem konnten ein Paar interessante Beispiele diskutiert werden. Die Studierende müssten aus ihrer Sicht die richtige Auswahl treffen und begründen. Die zweite Aufgabe dient hier vielmehr für genauere Interpretation der Aussagen von Teilnehmern. Es lassen sich bestimmte Aspekte aus den Aussagen herauszufiltern, die einen überschaubaren Verständnis in die Konzeptvorstellungen der Probanden erlauben. An konkreten Beispielen werden nun einige Beobachtungen dargestellt und diskutiert.

Beispiel 1: Der Teilnehmer hat in der ersten Aufgabe die Antwort KZKZKZ gewählt und begründet: "Beide Ereignisse haben die gleiche Wahrscheinlichkeit daher ist bei mehreren Würfen gleichmäßige Verteilung am wahrscheinlichsten.» Bei der zweiten Aufgabe hat der Teilnehmer sich für die erste Antwortmöglichkeit $K$ entschieden mit Begründung aus der ersten Aufgabe und Anmerkung, dass «jetzt Zeit für $K$ wäre».

Hinter dieser Fehlvorstellung steckt ein Fehlverständnis über das Modell. Der Teilnehmer scheint den Konzept der Gleichwahrscheinlichkeit so verstanden haben, dass er diese nicht nur auf einzelne Ereignisse anwendet, sondern auch für längere Versuchsserien annimmt. Dies führt zur Vermutung, dass die auf sekundärer Basis gewonnene mathematische Vorstellung einfach neben der primären Intuition aus dem Alltag nebenbei existiert. Denn die Menschen haben ein intuitives Verständnis dafür, was zufällig ist und was nicht. Dieses Verständnis ist aber leider nicht immer richtig. Tatsächlich erwarten die Individuen von an und für sich zufälligen Vorgängen, dass sie sich möglichst ständig abwechseln.

Beispiel 2: Der Teilnehmer hat in der ersten Aufgabe die Antwort KKKKZK gewählt mit Begründung: «Nur weil für die beiden Kopf und Zahl die Wahrscheinlichkeit bei $50 \%$ liegt, bedeutet dies nicht, dass diese abwechselnd auftreten. Es ist eher wahrscheinlich das eine Serie öfter vorkommt, aber der Intervall auch durch die andere Seite unterbrochen wird.» Bei der zweiten Aufgabe hat dieser Teilnehmer die letzte Antwortmöglichkeit $K$ und $Z$ sind beide möglich ausgewählt. Seine Begründung lautet: " $K$ und $Z$ haben die gleiche Wahrscheinlichkeit, egal wie oft vorher $Z$ geworfen wurde.» Offensichtlich wurde hier der Aspekt der Gleichwahrscheinlichkeit verstanden, was die Antwort für die zweite Aufgabe bestätigt. Die Wahl in der ersten Aufgabe lässt jedoch vermuten, dass der Teilnehmer eine falsche Intuition zur Stabilisierung der relativen Häufigkeit gemäß des empirischen Gesetzes der großen Zahlen entwickelt hat. Im Zusammenhang mit dieser Überlegung ist bei dem Teilnehmer eher die Vorstellung über die Stabilisierung der absoluten Häufigkeit vorhanden, was allerdings nicht tragfähig ist.

Beispiel 3: Der Teilnehmer wählt in der ersten Aufgabe $K Z K Z K Z$ mit Begründung «50:50. Also sind $K$ und $Z$ sind abwechselnd wahrscheinlich.» Bei der zweiten Frage entscheidet er sich für $K$ und argumentiert «wie oben 50:50». Die 50:50 Aussage lässt vermuten, dass der Teilnehmer den Aspekt der Gleichwahrscheinlichkeit versteht, jedoch fixiert er diese ohne zu beachten, dass jeder Wurf von dem vorherigen unabhängig ist. Einerseits musste die zweite Aufgabe seine Vorstellung mit 50:50 stören und zum Widerspruch der vertretenen Auffassung führen, andererseits erscheint die Fixierung auf Gleichwahrscheinlichkeit so stark, dass die Unabhängigkeit der Ereignisse vollkommen ausgeblendet wird.

2.1.3. Konstruktive Grundvorstellungen. Diese drei Beispiele bilden den Ausgangspunkt für die Überlegungen zu konstruktiven Maßnahmen für den eigenen Mathematikunterricht. Somit ist der Übergang zu der konstruktiver Dimension der Grundvorstellungsebene gegeben. Hierbei muss zuerst geklärt werden, worauf sind die etwaige Divergenzen zurückzuführen. Wenn es in eigenem Unterricht passiert, kann die Lehrperson den Unterrichtsverlauf analysieren und eventuell ermitteln, wie es zu einer oder anderen Fehlvorstellung kommen konnte. Daraufhin muss man überlegen, wie sind die Unterschiede normativer und deskriptiver Dimension zu beheben, welche Maßnahmen eine Verbesserung des aktuellen Zustandes bewirken können. Mittlerweile gibt es viele didaktische Ansätze und Vorschläge zur Verbesserung stochastischen Denkens. Die Entscheidung welche Maßnahmen für Verbesserung der Begriffsverständnis konkret ausgewählt werden sollen, ist von der Lehrperson aber auch von dem Lerner abhängig.

Für den Teilnehmer aus dem 1. Beispiel lassen sich die Divergenzen eventuell mit dem Ansatz von Schrage (1984) beheben. Schrage geht in seiner Arbeit auf konkrete lokale Maßnahmen zur Korrektur von Fehlern ein und zielt speziell auf das Ersetzen unkorrekter Sekundärintuitionen durch korrekte ab. Für ihn ist es wichtig den Lernenden nicht nur zu zeigen, warum eine normative Lösung korrekt ist, sondern warum der Denkprozess fehlerhaft ist. Anschaulich hat es dann Bazerman (2006) erklärt: Im ersten Schritt muss dem Teilnehmer die kognitive Verzerrung klar sein. Dies kann über Reflexion und verschiedene Beispielaufgaben erfolgen. Im zweiten muss die korrekte Lösung erklärt werden, dieses führt zu Verankerung einer neuen Vorstellung und ist dann auch der dritte und abschließende Schritt. Zur Unterstützung dieses Ansatzes schlägt Schrage folgendes Vorgehen:

1. Verschiedene Lösungen und Begründungen miteinander vergleichen.

2. Simulation des Münzwurfs aus der Aufgabe 1 (Experiment, Computer).

3. Bewertung und Auswertung der Lösungen, Begründungen aus dem ersten Punkt und auch der Vergleich der Ergebnisse mit durchgeführter Simulation.

4. Identifizierung der fehlerhafter Vorstellung.

5. Die Ausarbeitung korrekter Lösung und Begründung.

Mit den anderen Beispielen kann man analog verfahren und sich für irgendeine erprobte Verbesserungsmaßnahme entscheiden. Dies wird in diesem Artikel nicht ausgeführt, da der Handlungsprinzip von der Lehrkraft aber auch von dem Lernenden selbst abhängt.

\section{SCHLUSSWORT}

Viele andere Artikeln (vgl. Hattermann 2015, Malle 2003, Vohns 2005, Wörner 2014) berichten über die mathematische Grundvorstellungen und auch die Fehlvorstellungen. Immer wieder werden neue Lösungen und Perspektiven entdeckt, entscheidend in diesem Artikel ist jedoch die Herangehensweise an die Kompetenzorientierung mit dem Modellierungskreislauf 
und dem Grundvorstellungskonzept. Das wichtigste dabei ist das mathematische Arbeiten als Prozess zu betrachten und in dem Grundvorstellungskonzept die Verbindung zwischen den einzelnen Grundvorstellungsdimensionen zu schaffen. Mit in diesem Artikel vorgeschlagener Herangehensweise gelingt es eventuell viel wirksamer für die Lehrkräfte die fehlerhafte Vorstellungen $z u$ erkennen, $z u$ interpretieren und demnach qualifiziert zu beheben.

\section{References}

1. Bazerrman, M.N. (1986). Judgment in Managerial Decision Making. New York: Wiley.

2. Blum, W., Hofe, R. v., Jordan, A., Kleine, M. (2004). Grundvorstellungen als diagnostisches und aufgabenanalytisches Instrument bei PISA. In: Neubrand, M. (Hrsg.): Mathematische Kompetenzen von Schülerinnen und Schülern in Deutschland. Vertiefende Analysen im Rahmen von PISA 2000. Wiesbaden: WS Verlag für Sozialwissen-schaften, 145-157.

3. Blum, W., Leiss, D. (2005). Modellieren im Unterricht mit der «Tanken» - Aufgabe. Mathematik lehren, 128, 18 - 19.

4. Freudentahl, H. (1983). Didactical Phenomenology of Mathematical Structures. Dordrecht: Reidel.

5. Hattermann, M. (2015) Grundvorstellungsumbrüche beim Übergang zur 3D Geometrie. In: M. Ludwig, A. Filler, A. Lambert (Hrsg.): Geometrie zwischen Grundbegriffen und Grundvorstellungen. Jubiläumsband des Arbeitskreises Geometrie in der Gesellschaft für Didaktik der Mathematik. Wiesbaden: Springer, 75-86.

6. Hofe, R.v., (1995) Grundvorstellungen mathematischer Inhalte. Heidelberg: Specktrum.

7. Kleine, M. (2004). Quantitative Erfassung von mathematischen Leistungsverläufen in der Sekundarstufe I. Hildesheim: Franzbecker.

8. Kleine, M. (2012). Lernen fördern: Mathematik in der Sekundarstufe I. Seelze: Friedrich Verlag.

9. Malle, G.; Malle, S. (2003). Was soll man sich unter einer Wahrscheinlichkeit vorstellen? Mathematik lehren, 118, 52-56.

10. 10. Schrage, G. (1984) Stochastische Trugschlüsse., in: Mathematica Didacta (7), 3-19.

11. Vohns, A. (2005). Fundamentale Ideen und Grundvorstellungen: Versuch einer konstruktiver Zusammenführung am Beispiel der Addition von Brüchen. Journal für Mathematikdidaktik, 26, 52-79.

12. Weinert Franz E. (2001) Vergleichende Leistungsmessung in Schulen - eine umstrittene Selbstverständ-lichkeit., in: Weinert Franz E. (Hrsg.), Leistungsmessungen in Schulen, Beltz, Weinheim, 17-31.

13. Wörner, D. (2014). Grundvorstellungen zum Flächeninhaltsbegriff ausbilden - oder Wie groß ist die Antarktis? In: E.M. Plackner, D. Wörner (Hrsg.): Grundlagen fördern. Materialien für den Mathematikunterricht 2. Hildesheim: Franzbecker, 151-186.

\section{БАЗОВА ОСВІТА ТА ОСНОВОПОЛОЖНІ ІДЕЇ - ЗРОЗУМІЛЕ ПОЄДНАННЯ МАТЕМАТИЧНИХ СТРУКТУР Неллі Шмельцер, Міхаель Кляйне \\ Білесельдський університет, Німеччина}

Анотація.

Формулювання проблеми. Наразі орієнтація на компетентність є важливою частиною дискусій про уроки математики. У таких дискусіях особливо важлива увага приділяється базовій математичній освіті. у цій статті ми обгрунтовуємо важливість математичної компетентності щодо математичної роботи та впроваджуємо математичну роботу як цикл моделювання. Основна увага приділяється процесам трансформації реальності у математику. Зокрема, трансформаційні прочеси сприяють кращому математичному розумінню учнів і тим самим сприяють покращенню якості викладання математики.

Матеріали і методи. Для досягнення наших цілей ми застосовуємо в чій статті емпіричні методи і загальні методи наукового пізнання: порівняльний аналіз для з'ясування різних поглядів на проблему та визначення напрямку дослідження, систематизація та узагальнення для формулювання висновків та рекомендацій, узагальнення авторського педагогічного досвіду та спостережень.

Результати. У главі 1.2 ми описуємо процес математичної роботи на різних етапах, використовуючи приклад типового завдання. Наведений приклад покликаний чітко розкрити процеси мислення та роботи відповідно до теоретичної моделі запропонованної у главі 1.1. Не слід також думати, що у загальній ситуації математична робота може бути вичерпно описана прикладом, який ми вивчаємо. Однак компетентнісно-орієнтована методика навчання застосовується для того, щоб студенти розробили нові стратегії та евристику для роботи з математикою як наукою. Для того, щоб учні розвивали свою математичну компетентність, потрібні ментальні моделі, які ми називаємо основоположними ідеями. Побудова таких пізнавальних структур називається формуванням основоположних ідей. Цей процес характеризується фіксачією значення нових термінів з точки зору відомих фрактичних зв'язків, побудовою ментальних об'єктів, що описують цей термін, та застосуванням їх у нових контекстах. Навчання включає як розширення, так $і$ зміну існуючих основоположних ідей, а також побудову нових ідей. Відповідно, глава 2.1 використовує приклад ймовірності, щоб проілюструвати, як різні аспекти ймовірності можна зрозуміти з точки зору такого фундаментального поняття і у який спосіб може відбуватися розбудова основоположних ідей. Суттєвим у цій статті є новий підхід, що орієнтується на компетентності з циклом моделювання та базовою концепцією основоположних ідей.

Висновки. У розробленому підході вказується на важливість розглядати математичну роботу як процес і створювати зв'язок між окремими рівнями основоположних ідей у базовій концепції. Використання запропонованної структури дає можливість вчителям більш функціональніше розпізнавати, інтерпретувати та відповідно вилучати з розгляду невірно осмислені основоположні математичні ідеї учнів.

Ключові слова: базові математичні ідеї, математична компетентість, цикл моделювання, трансрормація реальності, основоположні ідеї, ментальні об'єкти, ймовірність. 